\title{
The asymmetric radio remnant of SN 1987A
}

\author{
B. M. Gaensler \\ Australia Telescope National Facility, CSIRO, PO Box 76, Epping, NSW 2121, Australia and \\ Astrophysics Department, School of Physics A29, University of Sydney, NSW 2006, Australia. \\ Email: b.gaensler@physics.usyd.edu.au \\ and \\ R. N. Manchester, L. Staveley-Smith, A. K. Tzioumis, J. E. Reynolds and M. J. Kesteven \\ Australia Telescope National Facility, CSIRO, PO Box 76, Epping, NSW 2121, Australia
}

May 2, 2018

To appear in volume 479 of the The Astrophysical Journal (20 Apr 1997).

Received — 


\begin{abstract}
We present seven years of radio observations of SN 1987A made with the Australia Telescope Compact Array. At 1.4, 2.4, 4.8 and $8.6 \mathrm{GHz}$, the flux density of the radio remnant has increased monotonically since emission was redetected 1200 days after the explosion. On day 3200, the remnant was expanding at $2800 \pm 400 \mathrm{~km} \mathrm{~s}^{-1}$, which we interpret as indicating significant deceleration of the fastest moving ejecta. Since day 1787 the spectral index has remained constant at $\alpha=-0.95 \pm 0.04\left(S \propto \nu^{\alpha}\right)$. These observations are all consistent with the shock having encountered a denser, shocked, component of the progenitor's stellar wind. At the current rate of expansion, the shock is expected to encounter the inner optical ring in the year $2006 \pm 3$, in line with predictions made by hydrodynamic simulations.

Using super-resolution, we have also obtained $9 \mathrm{GHz}$ images of the remnant (resolution $\approx 0^{\prime \prime} .5$ ) at four epochs. The emission is distributed around the rim of a near-circular shell, but has become increasingly asymmetric with time. There are two "hotspots" to the east and west, aligned along the major axis of the optical ring. This morphology is most likely indicative of an axisymmetric circumstellar medium into which the shock is expanding, consistent with present understanding of the progenitor star and its environment. The two hotspots are increasing in flux density at different rates, which may indicate directional anisotropies in the ejecta. We believe that the northern and southern regions of the remnant are encountering a shocked wind which is less dense and also further from the progenitor star than that in the bright regions of emission. As a result, these regions should eventually brighten and/or extend.
\end{abstract}

Subject headings: supernovae: individual (SN 1987A) — supernova remnants — circumstellar matter 


\section{Introduction}

SN 1987A in the Large Magellanic Cloud has presented a unique opportunity. The nearest $(\sim 50 \mathrm{kpc})$ supernova $(\mathrm{SN})$ to have been detected since the invention of the telescope, its aftermath presents a detailed picture of its progenitor's circumstellar medium (CSM) and of the interaction of this CSM with the SN shock.

Since the discovery of radio supernovae (RSNe) over 25 yr ago (Gottesman et al. 1972), such objects have proven to be useful tools in probing the CSM of massive stars. The flux density from a typical RSN takes weeks to years to peak in its radio emission, then undergoes a slow power-law decay extending over a period of years or even decades (Weiler et al. 1996). This is representative of the SN shock sweeping up the dense, slow-moving wind generated by the red supergiant (RSG) progenitor (Chevalier 1982).

However, SN 1987A's progenitor, Sk-69²02, was very different. It is believed to have evolved from a RSG into a blue supergiant (BSG) approximately $2 \times 10^{4}$ yr before the SN event Crotts \& Heathcote 1991). During the star's period as a BSG, the high-velocity BSG wind interacted with the slower RSG wind surrounding it, creating a cavity. Ground-based and HST optical observations of SN 1987A have revealed a complex nebulosity surrounding the explosion, consisting of a central circular ring of diameter $\approx 1^{\prime \prime} .6(0.4 \mathrm{pc})$, and two similar rings of diameter $\approx 3^{\prime \prime} .6$ $(0.9 \mathrm{pc})$, one on either side. The whole system is inclined at $\sim 45^{\circ}$ to the line of sight Jacobsen et al. 1991; Plait et al. 1995). While the exact origin for this "triple ring nebula" is unclear, it appears to demarcate the interface between the BSG and RSG winds, material at the boundary being excited by the ultraviolet flash from the supernova (Luo \& McCray 1991). The optical emission appears to indicate that the BSG wind bubble is bipolar in shape (Crotts, Kunkel, \& Heathcote 1995), the "standard" model being that this is caused by an RSG wind which is denser at the equator than at the poles (Luo \& McCray 1991; Martin \& Arnett 1995). Problems exist with this model however (Blondin 1994; Burrows et al. 1995), and many alternatives have been proposed (e.g. McCray \& Lin 1994; Burderi \& King 1995). 
Radio emission from SN 1987A was detected at $0.843 \mathrm{GHz}$ by the Molonglo Observatory Synthesis Telescope (MOST) on 1987 February 25, two days after the SN event. This emission reached a maximum four days after the explosion (Turtle et al. 1987), then followed a power-law decay, to become undetectable less than a year later (Ball et al. 1995). This radio outburst is understood to be a consequence of the rapidly moving, low-density BSG wind, which produced only a short-lived period of radio emission when hit by the SN shock Storey \& Manchester 1987; Chevalier \& Fransson 1987).

After remaining quiescent for several years, radio emission from the remnant of SN 1987A was redetected by both the MOST and the Australia Telescope Compact Array (ATCA) in mid-1990 (Staveley-Smith et al. 1992, hereafter Paper I), approximately $1200 \mathrm{~d}$ after the explosion. At $9 \mathrm{GHz}$, the ATCA's highest frequency, the remnant was just resolved, and by early 1992 the emission was found to deviate by $\sim 10 \%$ from spherical symmetry (Staveley-Smith et al. 1993b), hereafter Paper II). Super-resolution of ATCA data at comparatively low signal-to-noise produced images of resolution $\approx 0^{\prime \prime} .5$ (Staveley-Smith et al. 1993a, hereafter Paper III). In an image corresponding to observations made in 1993 January, radio emission is distributed around the rim of a shell, with up to $50 \%$ of the flux density originating from two bright limbs on the shell's eastern and western edges. To account for the size of the remnant at the second radio turn-on, a velocity of $\sim 35000 \mathrm{~km} \mathrm{~s}^{-1}$ was required for the fastest ejecta. However, only a marginal increase in size was subsequently detected over a 20-month period, implying that the remnant had either decelerated significantly, or was rapidly changing shape. This emission, which has since been shown to be centred on the position of the SN (Reynolds et al. 1995), was still increasing in its intensity four years after its reappearance (Ball et al. 1995; Staveley-Smith et al. 1996, hereafter Paper IV).

The second onset of radio emission has been interpreted as resulting from the shock encountering a sudden increase in the density of the CSM. The diameter of the emitting region is noticeably smaller than the inner optical ring, and so the shock is yet to reach the undisturbed RSG wind. Chevalier (1992a) has argued that the shock has reached the termination shock of 
the BSG wind, so that it is now progressing through a region of constant density. Such a theory is supported by more detailed modelling of the radio emission (Ball \& Kirk 1992; Duffy, Ball, \& Kirk 1995, hereafter DBK). Chevalier \& Dwarkadas (1995, hereafter CD95), have recently proposed an alternative model, that the dense region that the shock has encountered is actually an ionized component of the $R S G$ wind located inside the optical ring. The greater density of this RSG component can better explain the appearance of X-ray emission from SN 1987A (Beuermann, Brandt, \& Pietsch 1994; Gorenstein, Hughes, \& Tucker 1994), and also the large apparent deceleration of the SN shock suggested by the results of Paper III.

Only two other remnants of supernovae (SNe) as young as SN 1987A have been sufficiently resolved at radio frequencies to reveal morphological details, namely SN 1986J (Bartel et al. 1991) and SN 1993J (Bartel et al. 1994; Marcaide et al. 1995a), both using VLBI (resolution < 1 mas). While these supernovae are very different from SN 1987A in their luminosities, radio light curves and properties of their progenitor stars (e.g. Ball \& Kirk 1995), the brightness distribution of both objects deviates from that of a uniform spherical shell, at least to some extent. Thus it is clear that SN 1987A is not unique in having an asymmetric radio morphology so early in its lifetime. However, SN 1987A is unique in that we have a considerable amount of information about the progenitor star and its environment which we can use to try and explain the observed radio structures. Also, SN 1987A appears be at a later, or at least different, stage in its evolution: the other two objects are still in the standard RSN phase, a phase which lasted just days in the case of SN 1987A.

Previous ATCA data published on SN 1987A consists of 4.8 and 8.6 GHz flux densities up to day 2300, and a single super-resolved $9 \mathrm{GHz}$ image. In this paper we present the entire ATCA dataset, to date, on SN 1987A. Flux density measurements have been carried out at intervals of 1-2 months at 1.4, 2.4, 4.8 and 8.6 GHz from day 918 (1989 August) up to day 3325 (1996 April). These seven years of observations cover the entire second phase of the radio evolution up to this point. We have also obtained $9 \mathrm{GHz}$ images of the remnant at four epochs from 1992 to 1995. With the increasing flux density of the remnant, these images are becoming progressively more 
reliable and detailed.

Such observations provide a key insight into the interaction of the SN shock with the CSM. The flux density of the remnant as a function of time and frequency is our best guide to conditions at the shock, such as the details of the acceleration mechanism and the properties of the material into which the shock is expanding. The radio images of the remnant demonstrate the non-spherical nature of the system, and can be interpreted in terms of the three-dimensional structure of the surrounding medium and of the explosion itself. From these data the true rate of expansion of the remnant can also be measured. The observations made in this project are discussed in Section 2 . Results are presented in Section 3, and are discussed in terms of the properties of SN 1987A and its surroundings in Section 4 .

\section{Observations}

All observations were made with the Australia Telescope Compact Array (Frater, Brooks, \& Whiteoak 1992), an aperture synthesis telescope consisting of six 22m-diameter antennae located on a $6 \mathrm{~km}$, east-west baseline near Narrabri, New South Wales. Two types of observations have been made of SN 1987A using the ATCA: monitoring and imaging. All observations were continuum observations, measuring two frequencies simultaneously each with a bandwidth of $128 \mathrm{MHz}$. All Stokes polarization parameters were recorded. Amplitudes were calibrated using the revised scale of Reynolds (1994), assuming a flux density for PKS 1934-638 of 15.0, 11.6, 5.8 and 2.8 Jy at frequencies of $1.4,2.4,4.8$ and $8.6 \mathrm{GHz}$, respectively $\left(1 \mathrm{Jy}=10^{-26} \mathrm{~W} \mathrm{~m}^{-2} \mathrm{~Hz}^{-1}\right)$. Flux density measurements in Papers I \& IV used an earlier scale; measurements at 1.4, 2.4 and 4.8 $\mathrm{GHz}$ were on a scale $8-12 \%$ higher, and those at $8.6 \mathrm{GHz} 10 \%$ lower, than values quoted here. Phases were calibrated using the source PKS 0530-727 and also, in conditions of poor phase stability, PKS 0454-810 (see Reynolds et al. 1995). 


\subsection{Monitoring}

An ATCA monitoring observation of SN 1987A normally gives a measurement of the flux density of the source at four frequencies. The ATCA can observe at two frequency bands simultaneously: earlier observations were at either $1.4 / 2.4 \mathrm{GHz}$ or $4.8 / 8.6 \mathrm{GHz}$, while more recent observations used frequency switching between these two pairs. The integration time for an observation was typically $6-12 \mathrm{~h}$.

Until 1995 May, monitoring data were reduced using the AIPS package. After this date, the Miriad package (Sault, Teuben, \& Wright 1995) was used. To check the consistency between these two, we reduced several observations using both packages. The corresponding difference in flux density was in all cases considerably less than the uncertainty.

Data were edited, calibrated and imaged. Baselines shorter than $3 \mathrm{k} \lambda$ were often contaminated by emission from strong sources outside the primary beam (primarily 30 Doradus), and were discarded. Images were then deconvolved using the CLEAN algorithm (Clark 1980). Before day 1500, the flux density of SN 1987A was equated to the peak brightness of the source. After this date, the flux density was measured by fitting a Gaussian profile to the emission.

\subsection{Imaging}

Imaging observations were made three times a year from 1992 to 1995. The three observations in a given year were always made with different $6 \mathrm{~km}$ configurations to give increased radial coverage in the $u-v$ plane. Each observation used two simultaneous frequencies, both between 8.6 and $9.1 \mathrm{GHz}$, giving a diffraction-limited synthesised beam of $\approx 1^{\prime \prime}$. Integration times were long $(12-16 \mathrm{~h})$ to give increased sensitivity. To avoid possible artifacts, the phase centre was placed $10^{\prime \prime}$ south of SN 1987A. More information on these observations is contained in Table 1 .

All imaging data were reduced using Miriad. Observations corresponding to earlier images (Paper III) were completely reprocessed. Data were carefully examined for bad points, and 
observations during which the atmospheric phase stability was poor were rejected. The data were then calibrated and the three sets of observations for each year combined. Images centred on the remnant were produced using multi-frequency synthesis (Sault \& Wieringa 1994), using a cell size of $0^{\prime \prime} .08$ in each coordinate and an image size of $512 \times 512$ pixels. As a compromise between natural and uniform weighting, Briggs' robustness parameter was used with a value of 0 (Briggs 1995). This suppressed stripes in the dirty map caused by the small cell size. Deconvolution was carried out using a maximum entropy (MAXEN) algorithm (Gull \& Daniell 1978) with 1000 iterations, using a square window of side $\approx 4^{\prime \prime}$ centred on the remnant. The MAXEN model was then convolved with two different restoring beams: the first was the diffraction-limited beam, the second was a super-resolved circular beam with FWHM $0^{\prime \prime} .4$ as used in Paper III. This model was then combined with the unscaled residuals, and then regridded at a pixel scale of $0^{\prime \prime} .01$ in each coordinate to produce the final image.

\section{Results}

\subsection{Monitoring}

Monitoring data for SN 1987A at all four frequencies are shown in Figure 1. Two uncertainties are associated with each measurement: a random error, representative of the noise in the image and the uncertainty in the fitting process, and a systematic error of order $5-10 \%$, estimated by examining the scatter in the flux density of a nearby $\left(\sim 4^{\prime}\right)$ unresolved source which we designate 0536-6919. T These errors are combined in quadrature to give the error bars shown in Figure 1.

The flux density at all frequencies has continued to increase essentially monotonically over the entire period of observations, and shows no sign of levelling off. A linear fit over the last two years of data gives a rate of increase of $33.5 \pm 3.1,23.0 \pm 3.0,10.9 \pm 1.4$ and $4.2 \pm 1.5 \mu \mathrm{Jy} \mathrm{day}^{-1}$ at

\footnotetext{
${ }^{1}$ This source is variable. However, the estimate of the systematic scatter in its light curve was made over a period of $\sim 400 \mathrm{~d}$ during which its flux density appeared to remain constant.
} 
1.4, 2.4, 4.8 and $8.6 \mathrm{GHz}$, respectively. Short-time-scale variations are evident in the data - the significance of these is discussed in Section 4.1.2.

Spectra for SN 1987A at five epochs are shown in Figure 2. These observations are consistent with a constant spectral index $\alpha=-0.95 \pm 0.04$ over more than four years.

\subsection{Imaging}

\subsubsection{Total intensity}

Two sets of total-intensity images are shown in Figures 3 through 6: first the diffractionlimited images, then the super-resolved images obtained by using a circular, $0^{\prime \prime} .4$ FWHM restoring beam. Only the inner $\approx 4^{\prime \prime}$ of each $40^{\prime \prime}$ image is shown, and no emission above the noise was seen outside this central region. More information on each image is given in Table 1. Figure 7 shows an SN 1987A difference image, produced by subtracting the super-resolved image made in the 1993 epoch from that made in 1995.

In the diffraction-limited images of the supernova, the source is clearly resolved. There is a distinct asymmetry associated with the emission, in that the peak is displaced east of centre. While the object increases in brightness with time, it does not appear to be getting noticeably larger - this is quantified in Section 3.2.3.

The super-resolved images show that, rather than being centrally peaked, the emission is restricted to the rim of an approximately circular shell. The 1992 image is (unsurprisingly) similar to that produced in Paper III: two lobes are apparent on the east and west limbs of the remnant. In the 1993 and later images, the remnant continues to brighten, and takes on a more circular shape. For the 1995 epoch, the minimum of emission at the centre of the remnant is $330 \mu \mathrm{Jy}$ beam $^{-1}$, well above the noise, and this can be considered an upper limit on the flux density of a central point source. Using the CLEAN algorithm produces qualitatively similar images, although it is worth noting that CLEAN is not recommended for super-resolution (Briggs 1994). 
The difference image in Figure 7 shows the two lobes or "hotspots" increasing in their intensity, the eastern hotspot clearly increasing in brightness more rapidly than the western one. In 1992 the ratio of peak brightness between the east and west lobes was 1.2, but by 1995 it was 1.8. Approximately $50 \%$ of the total flux density from the remnant is contained in these regions. In all images, the two hotspots are at consistent position angles of $\sim 100^{\circ}$ and $\sim 260^{\circ}$. The range in position angle of the diffraction limited beams in Table 1 is large, and indeed the beams for the 1993 and 1994 epochs are nearly circular. This is good evidence that the consistent orientation of the hotspots observed in the succession of super-resolved images is not an artifact driven by the $u-v$ coverage of the observations. Further tests on the reliability of such position angles under super-resolution have been carried out by Briggs (1995).

In the 1992 image, there appears to be an extension of emission towards the north of the remnant. This vanishes in later images and is likely to be an artifact introduced by super-resolution under conditions of low signal-to-noise ratio (cf. Briggs 1994). In the 1994 and 1995 images, the eastern hotspot seems to extend towards the south, and the difference image in Figure 7 indicates that this may be a weaker, separate hotspot forming on the southern rim of the remnant.

Super-resolution is, in effect, an extrapolation of the $u-v$ data out to projected interferometer spacings beyond those measured by the telescope. However, the long integration times, extensive $u-v$ coverage obtained and the simplicity of the structure in the resultant images justify a moderate increase in resolution over that dictated by diffraction theory (Narayan \& Nityananda 1986). The consistency of the results further adds to the credibility of the method: in 12 (three for each year) independent datasets, each with different $u-v$ coverage and with differing noise levels, the same features are reproduced in each of Figures 3 through 6 - a roughly circular shell, with bright lobes to the east and west. For comparison, corresponding images of 0536-6919 are shown in Figure 8. These show that for a simple unresolved source, super-resolution behaves as expected, producing a Gaussian region of emission with a reduced half-width and with an unchanged total flux density. A more in-depth study on the reliability of super-resolved images is described by Briggs (1994, 1995). 
Figure 9 shows a comparison between the super-resolved radio image from 1995 and a WFPC2 HST image of the central source and the surrounding inner optical ring (Burrows et al. 1995). The images were aligned by first establishing an accurate position for the central source in the Hipparcos optical reference frame, and then linking this with the radio reference frame as determined by VLBI (Reynolds et al. 1995). The residual uncertainty between the VLBI reference frame and the ATCA image is $\sim 0^{\prime \prime} .05$. The position angles of the two opposed brightness enhancements in the radio image are similar to that of the major axis of the optical ring $(\mathrm{PA} \approx$ $\left.81^{\circ}\right)$. Although the minimum in the radio emission aligns closely with the central source, this is not so in earlier images, and is not considered significant.

\subsubsection{Polarization}

Images were formed in the Stokes parameters Q, U and V, deconvolved using the CLEAN algorithm and restored with a diffraction-limited beam. A linear polarization, L, image, was formed by combining Q and U. In the 1992, 1993 and 1994 data, no linear polarized emission was detected from the radio remnant. In the 1995 data, there is a marginal $(2 \sigma)$ detection from a region in the southwest of the remnant, corresponding to a total fractional polarization of $\sim 0.01 \%$. No circularly polarized emission was detected at any epoch.

\subsubsection{Expansion of the remnant}

Using the method described in Paper II, we obtain an estimate for the size of the radio remnant at each epoch by fitting a two-dimensional projection of a thin, spherical shell to $9 \mathrm{GHz}$ $u-v$ data. We have used all the data shown in Figure 2 of Paper III, as well as that in Table 1 . The results of the model fitting are shown in a plot of radius versus time in Figure 10. For the most recent (1995 November) data, a radius of $r=0^{\prime \prime} .685 \pm 0^{\prime \prime} .005$ is obtained, $84 \%$ of the radius of the optical ring (Burrows et al. 1995).

A power-law fit to the data of the form $r / r_{0} \propto\left(t / t_{0}\right)^{m}$ (where $t$ is the time since the SN 
explosion and $t_{0}=1800 \mathrm{~d}$ ) gives $r_{0}=0^{\prime \prime} .641 \pm 0.006$ and $m=0.12 \pm 0.02$. At day 3200 , this

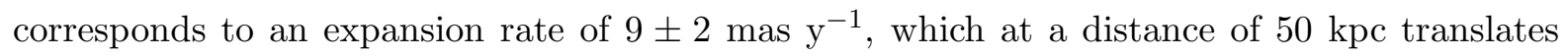
to $2200 \pm 400 \mathrm{~km} \mathrm{~s}^{-1}$. The value obtained for $m$ is slightly less, but consistent with, the value $m=0.17 \pm 0.08$ obtained using data up to day 2100 (Paper III).

A power-law fit implies that the shock is currently decelerating. However, the data are equally well fitted by a straight line. This line does not pass through the origin, indicative of a rapid deceleration in the past but minimal deceleration in the present. Such a fit gives an expansion rate of $12 \pm 2$ mas $^{-1}\left(2800 \pm 400 \mathrm{~km} \mathrm{~s}^{-1}\right)$. This is the value used in subsequent discussion.

Modelling directly in the $u-v$ plane is a robust and consistent way of quantifying the relative size of the remnant as a function of time, and the results are not complicated by issues of deconvolution and resolution. However, it is clear that as time progresses, the approximation of a thin spherical shell becomes less and less justified, and measurements of the angular size may be skewed by the changing appearance of the remnant. Therefore, to confirm these results, the size of the remnant was also measured directly in the image plane.

For each super-resolved image of SN 1987A, the radius of the remnant was measured along lines joining the position of the central optical source (Reynolds et al. 1995) and the northern maximum, southern maximum, eastern saddle and western saddle, out to the point where the intensity falls to $50 \%$ of its value at the respective maximum or saddle. These measurements show no measurable expansion in any direction, with an upper limit of $\sim 7000 \mathrm{~km} \mathrm{~s}^{-1}$. Although this method is somewhat crude, it is consistent with the result obtained from the $u-v$ fitting above.

We find that the eastern side of the remnant is $\approx 130 \pm 70$ mas further from the central source (in projection) than the other components. Reynolds et al. (1995) noted a similar displacement by comparing the centre of symmetry of the 1992 epoch image to the position of the central source.

\section{Discussion}




\subsection{Flux density}

\subsubsection{Long-term behaviour}

The second turn-on in radio emission from SN 1987A (Paper I) has generally been understood as being caused by the shock encountering a sudden increase in the density of the CSM, presumed to be the reverse shock of the BSG wind (Chevalier 1992b; Beuermann, Brandt, \& Pietsch 1994). Blondin \& Lundqvist (1993) point out the possibility that no reverse shock exists, so that radio emission must somehow be generated by density enhancements of unknown origin in the free wind. However, the good fit to the radio light curve produced by both simple (Paper IV) and detailed (DBK) models, and the good evidence presented in Sections 3.2 .3 and 4.2 for deceleration, are both strong arguments in favour of the shock having encountered a region of increased density.

Models of such an encounter with the shocked BSG wind predict that the flux density should level off or begin to decrease (Chevalier 1992b; DBK). Yet it is clear from Figure 1 that the flux density continues to rise. The ellipsoidal geometry of the BSG wind cavity may be a contributing factor, in that the distance to the density jump is greatest along the symmetry axis of the progenitor system (Blondin \& Lundqvist 1993). The radio flare produced by the encounter of the shock with dense material is consequently spread out over a greater period of time before decaying. However, the morphology of the remnant suggests that emission along this axis does not contribute significantly to the total flux (see further discussion below in Section 4.4.3). Another possibility is that the presence of toroidal magnetic fields within the BSG wind-bubble serves to boost the radio emission (Chevalier \& Luo 1994). This model can probably be ruled out though by the low level of linear polarization observed, which argues against the presence of magnetic fields with such a well-defined geometry (Section 4.3 below). A recently proposed alternative is that the shock has encountered an ionized region of the RSG wind located inside the optical ring (CD95). This Hil region is calculated to be $\approx 400$ times more dense than the stagnation zone of the BSG wind. The large consequent deceleration experienced by the shock, combined with the increased particle injection, could sustain the radio flux density as is observed. 
We do not attempt to model the radio light curves, other than to note that the simple model of Paper IV for flux densities up to day 2300 still fits the data to a reasonable extent over the increased time period. The morphology of the remnant is becoming increasingly asymmetric with time - not only are there two strong hotspots, but they are brightening at different rates, and new features may be forming. Thus it is clear that the assumption made in earlier analyses of a spherical emitting region (DBK; Paper IV) is no longer valid, and that more complex two- or three-dimensional modelling is now required. Such a study is beyond the scope of this paper.

\subsubsection{Short-term behaviour}

In the ATCA data presented in Figure 1, small-scale fluctuations in SN 1987A's flux density are apparent at all four frequencies over timescales of $\sim 100 \mathrm{~d}$ (comparable to the spacing between observations). This is in contrast with MOST data, for which Ball et al. (1995a) conclude that there have been no variations of amplitude $>2 \%$ on timescales between 30 and a few hundred days. In Paper I, $4.8 \mathrm{GHz}$ variations were reported at the level of $\sim 20 \%$ over a two-day period, six months after the radio emission reappeared. However, since then the sampling interval has been far coarser, and we are unable to determine if such short-period variability is still occurring.

The nature of the ATCA means that flux densities are not as accurate as those measured by the MOST. While the MOST has complete coverage in the $u-v$ plane over a full 12 -h synthesis and obtains all spatial frequencies from $43 \lambda$ to $4415 \lambda$, the ATCA has just 15 baselines spread over $6000 \mathrm{~m}$. In addition, frequency switching and phase calibration means that only about a third of the time can be spent integrating on SN 1987A at a given frequency. These effects produce large sidelobes in the resultant images. Figure 1 suggests that flux density variations become more pronounced at higher frequencies. However, this is likely the result of atmospheric phase variations which, at 4.8 and $8.6 \mathrm{GHz}$, can cause the signal to decorrelate. There is the suggestion of some correlations between frequencies which were observed simultaneously $(1.4 / 2.4 \mathrm{GHz}$ or 4.8/8.6 GHz), but the factors discussed above will affect the data at both such frequencies similarly. We conclude that the small-scale changes in flux density seen in ATCA data are probably not 
significant — the light curve obtained by MOST is far more reliable.

\subsubsection{Spectral index}

Observations in the first year after the radio emission re-appeared showed significant variations in the spectral index (Paper I). Correcting for the ATCA revised flux density scale Reynolds 1994), the spectrum flattened from $\alpha<-1.6\left(S \propto \nu^{\alpha}\right)$ on day 1243 to $\alpha=-0.91 \pm 0.05$ on day 1517, before possibly steepening to $\alpha=-0.99 \pm 0.04$ on day 1595 .

The data in Figure 2 show that within the uncertainties, the spectrum did not vary from day 1787 (1992 January) to day 3325 (1996 April). The average value over this period is $\alpha=-0.95 \pm 0.04$, consistent with the value at day 1595 (1991 July).

In the model of DBK, the shock compression ratio, $\rho=1-3 / 2 \alpha$, begins at its maximum value of 4 when the explosion occurs, and consequently decreases because the shock is modified by the acceleration of cosmic rays. The compression ratio then lies on a plateau $(\rho \approx 2.7)$ for an extended period before then rapidly decreasing again. In DBK's standard model, the plateau phase lasts until approximately day 2000, and by day $\sim 3000, \rho<2$ ( $\alpha$ steeper than -1.5 ). However, observations imply that on day 3325, $\rho=2.58 \pm 0.11$. For comparison, the spectrum on day 1517 corresponds to $\rho=2.65 \pm 0.14$.

So, while we indeed observe a plateau, our results indicate that this phase is considerably more extended than predicted, and that accelerated protons are yet to smooth out the shock completely. In DBK's model, the behaviour of $\rho$ as a function of time depends on the distance from the progenitor star of the density jump responsible for the radio emission. DBK's Figure 7 shows that if this jump is moved further from the star, the plateau region does become more extended. The model of DBK assumes that the density jump encountered is the termination shock of the BSG wind. However, if, as discussed in Section 4.1.1, the cause of the radio emission is an ionized region of the RSG wind, the greater distance of this component from the progenitor can, in the context of this model, explain the constant spectral index. CD95 roughly estimate that the 
contact discontinuity with the RSG wind is $20 \%$ further from the star than the termination shock of the BSG wind.

The increasingly asymmetric morphology of the remnant implies that a direct interpretation of the spectral index in terms of a single shock compression ratio may be overly simplistic. For example, because structural information is only available at $9 \mathrm{GHz}$, we are unable to determine if different regions in the remnant have different spectra, as is observed in SN 1993J Marcaide et al. 1995b). If this is the case, an explanation for the observed constancy of the spectral index may require further insight.

\subsection{Expansion of the remnant}

Between the SN event in 1987 February and the second radio turn-on in mid-1990, a mean expansion rate can be calculated for the remnant of $\sim 35000 \mathrm{~km} \mathrm{~s}^{-1}$. This is consistent with free expansion since the explosion (Hanuschik \& Dachs 1987). However, by 1993 January, the remnant appeared to be expanding at only $4800 \pm 2300 \mathrm{~km} \mathrm{~s}^{-1}$ (Paper III). While this seemed to indicate considerable deceleration, there was only a 20-month period over which measurements of the remnant's size had been made, and the low signal-to-noise ratio meant that the errors in these measurements were large. Also, an image was available at only one epoch, so that the possibility that the remnant was rapidly changing shape could not be ruled out.

However, over a period three times longer than previously possible, a low expansion velocity

of $2800 \pm 400 \mathrm{~km} \mathrm{~s}^{-1}$ is still obtained. The succession of images demonstrates that the shell does not appear to be changing in its gross form - we therefore conclude that the explanation in Paper III for the observed deceleration is incorrect, and that the expansion speed obtained is realistic.

One explanation for the extremely low expansion velocity is that the emission observed is not from the shock at all, but from stationary or slowly moving clumps of material in the BSG wind. In this model, the shock is now considerably further from the star than the remnant we observe. However, the emission continues to increase at all radio frequencies, and the spectral index is not 
steepening. Both these observations are difficult to explain if electrons are not continuing to be accelerated in the emitting regions. We therefore favour the alternative, that emission from the radio shell traces the expanding shock front.

The fastest ejecta contain only a small fraction of the total mass. For example, in the model of Luo, McCray \& Slavin (1994), the ejecta have a density profile $n \propto v^{-9}$ for $v>4000 \mathrm{~km} \mathrm{~s}^{-1}$. Thus it might be argued that while the fastest ejecta are responsible for the radio turn-on at day 1200 (and have since been decelerated), the main body of slower moving material has now caught up with this material, and that it is the relatively unimpeded expansion of these ejecta which we are now observing.

In a simple calculation, $v$, the mean velocity from day 0 to day $t$ of ejecta currently at radius $r$, is given by

$$
v=18.6 \times 10^{3} \mathrm{~km} \mathrm{~s}^{-1}\left(\frac{r}{0^{\prime \prime} .687}\right)\left(\frac{t}{3200 \text { days }}\right)^{-1}
$$

where $r=0^{\prime \prime} .687$ is the angular size of the remnant at $t=3200 \mathrm{~d}$, using the linear fit in Figure 10 . Since in reality ejecta may have decelerated in reaching a radius $r, v$ is a lower limit on the initial velocity of the ejecta currently at this distance.

From Equation (1), we see that while the initial radio turn-on $\left(r \approx 0^{\prime \prime} .5, t \approx 1200 \mathrm{~d}\right)$ requires ejecta of velocity $\sim 35000 \mathrm{~km} \mathrm{~s}^{-1}$, emission from a thick shell at day 3200 still requires ejecta for which $v=10000-20000 \mathrm{~km} \mathrm{~s}^{-1}$. The consequence of this is that most of the mass ejected by the supernova cannot yet have reached the radio emitting regions - the remnant is the result of rapidly moving material which was significantly decelerated at some time in the past, presumably around the time that radio emission was re-detected. Figure 10 is consistent with minimal deceleration from day 1500 to day 3500, but we cannot rule out the possibility that deceleration continues to take place.

This apparent deceleration would appear to be the best observational evidence yet that the shock has encountered a marked increase in density. CD95 argue that this deceleration is further evidence for an encounter with the dense, ionized RSG wind. From their model, we calculate 
a mean velocity for the shock while it is in this region of $6100 \pm 1200 \mathrm{~km} \mathrm{~s}^{-1}$. If the remnant continues to decelerate, a still denser component may be required to explain the observations.

When the fastest ejecta reach the circumstellar ring, the system is expected to become a strong source of optical and UV line emission, and the X-ray and radio luminosity should show a rapid increase (Luo \& McCray 1991; Luo et al. 1994 ). This has clearly not yet occurred, and limits even the fastest ejecta to a mean velocity of $\approx 22000 \mathrm{~km} \mathrm{~s}^{-1}$ from day zero to day 3200 . Assuming that the remnant continues to expand at its current rate, our results indicate that the shock will reach the ring in the year $2006 \pm 3$, which agrees with the prediction of 2003 from X-ray observations (Hasinger, Aschenbach, \& Trümper 1996). This is also consistent with hydrodynamic models (e.g. Suzuki, Shigeyama, \& Nomoto 1993; Luo et al. 1994), the most recent estimate being 2005 \pm 3 (CD95). However, further deceleration of the ejecta could delay the encounter considerably.

In Section 3.2.3, we suggested that the eastern region of the remnant may be further from the centre of the explosion than other regions. If this is the case, then this region either had the highest initial velocity, or has been decelerated the least. That it is also the brightest region favours the former. This could represent anisotropy in the explosion, as discussed below in Section 1.4.2.

\subsection{Polarization}

The radio emission from the remnant is believed to be synchrotron emission (Ball \& Kirk 1992; Paper IV), and so is expected to be associated with high degrees of linear polarization. However, the remnant is comparable in size to the synthesised beam of the telescope, and so there may exist variations in position angle of the electric field vectors over spatial scales smaller than this beam. In addition, the projected direction of the magnetic field could also be varying along the line of sight. Both these effects can smear out any linear polarization and give a low value for L. From the low level of polarization observed, we can conclude that the magnetic field is disordered on a scale of $\sim 0.5^{\prime \prime}(0.1 \mathrm{pc})$, and certainly is not well organised within each hotspot. 


\subsection{Morphology of the radio emission}

It is well established that the triple-ring optical nebula surrounding the supernova has cylindrical symmetry, with the axis inclined at $\approx 43^{\circ}$ to the line of sight and at a position angle of $\approx-9^{\circ}$ on the plane of the sky (Jacobsen et al. 1991; Burrows et al. 1995; Plait et al. 1995). The supernova event itself also appears to have had a similar symmetry, the envelope being extended along the axis of the cylinder (Karovska et al. 1989). It is generally accepted that the axisymmetric geometry observed coincides with the axis of rotation of the progenitor star, or possibly with the axis of a progenitor binary system.

An optically thin cylindrical shell of radio emission inclined at $\sim 45^{\circ}$ to the line of sight will show two brightness enhancements on the inclination axis. Thus in Figure 9, the alignment of the two opposed radio hotspots with the major axis of the optical ring argues that, to first order at least, the radio emission has the same symmetry as the optical nebula. However, the enhanced brightness from the eastern limb of the radio remnant breaks this symmetry, and indicates that the true geometry is still more complex. Models of the flux density cannot distinguish between ring and spherical geometries for the radio emission (Ball et al. 1995b). However, the non-zero level of emission in the centre of the radio shell argues that, unlike the inner ring, the remnant is quasi-spherical and extended in three dimensions.

DBK attribute the anisotropies observed in the 1992 image to minor variations in conditions at the shock, and assume that the system is essentially spherically symmetric. However, the persistence of these anisotropies, and the increase of the asymmetry with time, argue that the non-spherical nature of the morphology is significant. Bartel et al. (1991) suggest three explanations for the asymmetries observed in the radio remnant of SN 1986J: a central pulsar could be affecting the morphology in some way, the explosion may have been asymmetric, or the CSM into which the shock is expanding is not isotropic. Each of these could also influence the evolution of SN 1987A, and we consider the three possibilities in turn. 


\subsubsection{Effect of a central pulsar}

Neither pulsed emission (e.g. Manchester \& Peterson 1996) nor a central compact source (Paper I) has yet been detected in the remnant of SN 1987A. Although the detection of neutrinos from the initial explosion suggests that a neutron star was formed, it may have undergone subsequent collapse to form a black hole (Chevalier 1992a). If this is not the case, effects such as luminosity, beaming, scattering and absorption may still prevent the detection of pulsed emission (Manchester 1988).

While the radio emission observed is clearly not "plerionic", it has been proposed that the formation of two opposed regions of enhanced emission in shell supernova remnants (SNRs) can be explained by jets or cones of emission from a central neutron star (Manchester 1987; Willingale et al. 1996). However, the lack of radio emission for several years between the SN event and the second turn-on, the steep spectral index, and the increasing asymmetry between the eastern and western hotspots cannot be explained by this model in its simplest form, and we consider it an unlikely explanation for the radio emission. It is worth noting that a model involving a pulsar with precessing jets has been proposed to explain the optical triple-ring nebula (Burderi \& King 1995).

\subsubsection{An asymmetric explosion}

There is reasonable evidence that supernova ejecta are not distributed isotropically. Models of SNe involving rotating progenitor stars produce excess ejecta in the equatorial plane (BisnovatyiKogan 1971; Bodenheimer \& Woosley 1983), and there is observational evidence to suggest that this can influence the morphology of an evolved SNR (Tuohy, Clark, \& Burton 1982; Kesteven \& Caswell 1987).

For a spherically symmetric ambient medium with no large-scale magnetic fields, the radio emission from the remnant will peak where the shock is strongest. Indeed, several authors have argued for a spherically symmetric BSG wind (e.g. Martin \& Arnett 1995) and the lack of polarization detected in the radio emission indicates that the magnetic field is disordered on 
small scales. With such simplifying assumptions, the alignment of the radio morphology with the presumed equatorial plane of the progenitor star suggests that the ejecta were predominantly toroidal. In addition, the shell is faintest in the north and the south, as would be expected if ejecta were primarily generated in the equatorial plane.

There are problems with this conjecture, however. First, the suggestion that a third, southern, hotspot may be forming in the shell (as shown in Figure 7) is difficult to explain. Second, the assumption that the CSM which the shock is encountering is spherically symmetric may well be a naive one, as discussed further in Section 4.4.3. Finally, although there is much evidence from both optical polarimetry and asymmetry in the line emission from the supernova to suggest that the envelope and ejecta were axisymmetric (Cropper et al. 1988; Méndez et al. 1988; Karovska et al. 1989; (Pun et al. 1995), most observations have extensions being produced predominantly in the polar directions. This is at odds with the equatorial dominance required here. However, the asymmetries represented in optical observations may only be of very low mass (e.g. Utrobin, Chugai, \& Andronova 1995) and may not be representative of the overall shock front. VLBI observations of SN 1993J have indeed shown that early measurements of asymmetry in the SN event need not translate to the morphology of the radio shell (Marcaide et al. 1995a). Also, simulations of axisymmetric ejecta show that the resulting shock front is almost spherical (Blondin, Lundqvist, \& Chevalier 1996). Given these results, we cannot completely rule out this hypothesis.

Rather than producing a toroidal ejection of material, SNe can also be directional in their anisotropy, as evidenced by the kicks imparted to neutron stars (Kaspi et al. 1996; Petre, Becker, \& Winkler 1996). In Section 3.2.3, it was shown that the eastern limb may have expanded slightly further than the western limb, which may be the result of more rapidly moving material being ejected in this direction. Note that optical spectroscopy of SN 1987A also gives evidence for such an event (Stathakis 1996). 


\subsubsection{Asymmetries in the CSM}

The nature of the triple-ring nebula surrounding SN 1987A is not completely understood (Burrows et al. 1995; Burderi \& King 1995; Panagia et al. 1996). However, it is generally agreed that its existence is consistent with the mass-loss history of the progenitor star Sk-69²02. Several authors have proposed as an explanation the interaction of an equatorially enhanced RSG wind with a spherically symmetric BSG wind (Luo \& McCray 1991; Wang \& Mazzali 1992; Martin \& Arnett 1995; CD95). Other possibilities include a spherically symmetric RSG interacting with an axisymmetric BSG (Blondin 1994), the effect of a binary companion (Podsiadlowski, Fabian, \& Stevens 1991; Lloyd, O'Brien, \& Kahn 1995) and the presence of a proto-stellar molecular disk (McCray \& Lin 1994). In any case, it is accepted that the surrounding CSM is not spherically symmetric.

Chevalier \& Luo (1994) have argued that the onset of radio emission from SN 1987A can be explained by toroidal magnetic fields in the BSG wind bubble. Only a slight enhancement in the magnetic field of the equatorial regions above the poles is required to produce the observed morphology (DBK). But as discussed in Section 4.3, the lack of observed polarization makes this explanation unlikely.

A plausible explanation for the hotspots observed in the radio emission is that these parts of the shock have simply encountered denser regions of the CSM than the fainter regions. This could be because the transition to the shocked wind occurs closer to the star in the bright regions, or because the density of the shocked wind varies with polar angle. These two possibilities are not mutually exclusive, and we consider the implications of each below.

If the termination between the free and shocked winds is not spherical, but is extended along the symmetry axis (Blondin \& Lundqvist 1993; CD95), the equatorial regions of the SN shock encounter the denser, shocked wind before the polar regions do. The polar regions can also be expected eventually to reach the shocked component of the wind, at which time the remnant might begin to take on a more uniform brightness distribution. The suggestion in the 1994 and 1995 
super-resolved images (Figures 5 and 6) and in the difference image (Figure 7) that the southern rim of the radio shell is now beginning to brighten may indicate that this is already happening.

The fact that the eastern hotspot is brighter than its western counterpart can be understood if this side of the expanding remnant reached the density jump first. This may correspond to the two-clump model of DBK, where the SN shock encounters two clumps of material with a $\sim 120$ d delay between them. No third jump in the light curve is seen which might correspond to the formation of a new hotspot to the south (A.J. Turtle, private communication), although its contribution to the total flux density would be low. This difference between the eastern and western sides of the remnant can be explained in terms of a break in the cylindrical symmetry of the surrounding CSM, although this could be at odds with the high degree of symmetry observed in the optical emission (Burrows et al. 1995). Light travel times may also have an effect (Ball et al. 1995b), but a more appealing prospect is that it is a result of the possible directional anisotropy in the initial explosion discussed in Section 4.4.2. If higher velocity ejecta were produced on the eastern side of the remnant, this can also explain why the eastern hotspot continues to brighten more rapidly than that in the west.

While the shocked wind is believed to have a constant density in the radial direction (e.g. DBK), it has been argued that the density may well vary with polar angle (Blondin 1994), so that even after all parts of the SN shock have reached the denser component of the wind, particle injection may be greatest around the equator. Such an axisymmetric density distribution in the CSM should produce extensions in the north-south direction (Paper III; Blondin et al. 1996). Although such extensions are clearly not observed, Blondin et al. estimate that protrusions along the poles only become significant $\sim 10$ yr after the explosion, and so may not necessarily be expected to occur at the current time.

It is worth noting that the amount of anisotropy in the CSM required to produce the observed morphology is low. Around any given circumference on the shell in the 1995 super-resolved image (Figure 6), the ratio of maximum to minimum brightness is no more than three. Using Equation (4.7) of Paper IV, this corresponds to a ratio of the ambient density between the 
equatorial and polar regions of $\sim 1.7$. Most models can easily account for such a difference, even if the shock is yet to reach the densest regions (Luo \& McCray 1991; Blondin \& Lundqvist 1993).

\subsubsection{Comparison to other RSNe and SNRs}

SN 1986J, one of the most luminous RSNe known, was imaged in 1988 by Bartel et al. (1991). It was then of comparable age to SN 1987A, and also had a radio remnant quite similar in morphology; it too had two opposed hotspots, one brighter than the other, superimposed on a rough circular shell. SN 1986J also has several protrusions extending beyond the shell. These appear to extend from local minima in the rim, and might be representative of the features discussed by Blondin et al. (1996) which are produced by the expanding SN shock in the less dense, polar, regions of an axisymmetric progenitor wind. As noted above, such features may, with time, become apparent in SN 1987A.

Marcaide et al. (1995b) present a time sequence of images of the remnant of SN 1993J, taken between 6 and 18 months after the explosion. Unlike SN 1987A, clear expansion has been detected

for this object - an expansion index of $m \approx 0.9\left(r \propto t^{m}\right)$ has been measured right from day zero, indicating minimal deceleration. At $8.3 \mathrm{GHz}$, the shell of SN 1993J is almost circular, and has relatively small (compared to SN 1987A) brightness variations around its circumference. Marcaide et al. (1995a) attribute these variations to small inhomogeneities in the CSM, possibly produced by a binary companion to the progenitor.

In SN 1993J, we have a remnant which has so far undergone minimal deceleration, and which is also reasonably uniform in its shape and brightness. If, from this, we argue that it is the same physical process which causes both the deceleration of a shock front and a non-uniform appearance for the radio remnant, then we are led to the conclusion that it must be a non-uniform CSM which gives SN 1987A its appearance.

The connection between radio supernovae and evolved supernova remnants is unclear Weiler et al. 1986; Wilkinson \& de Bruyn 1990), and there is the intriguing possibility that having long 
ago passed through its RSN stage, SN 1987A may be, as suggested in Paper III, in the very early stages of an evolution corresponding to that of a classical SNR. Manchester (1987) has argued that the passage of the SN shock through the progenitor wind, as is occurring in the case of SN 1987A, can "imprint" a remnant with a morphology which it retains through to an evolved state. For example, the small-scale symmetry of SNR G296.5+10.0 is indicative of the remnant's being shaped by events early in its lifetime (Storey et al. 1992). However, it is clear that the large-scale ISM (Landecker et al. 1982; Braun \& Strom 1986), as well as possibly large-scale magnetic fields (Roger et al. 1988; Fulbright \& Reynolds 1990), may both have a large effect on a remnant's morphology.

At low resolution, the bipolar, but asymmetric, appearance of older supernova remnants such as SNR G308.8-0.1 and SNR G320.4-1.2 (Whiteoak \& Green 1996) is suggestive of the morphology of SN 1987A. Such a comparison is, at least for now, pure speculation, although continued observations of SN 1987A over the next decade and beyond may give hints as to what this object might one day become.

\section{Conclusion}

In this paper, we have reported on all ATCA observations of SN 1987A from 1989 up to mid-1996, with the following main results:

1. The total flux density has increased monotonically since radio emission was redetected in 1990.

2. The spectral index has remained constant since 1992 January at $\alpha=-0.95 \pm 0.04$.

3. Assuming that the observed radio emission traces the shock, the remnant is the product of initially rapidly moving $\left(v=10000-35000 \mathrm{~km} \mathrm{~s}^{-1}\right)$ ejecta, which have subsequently

decelerated to $v \approx 3000 \mathrm{~km} \mathrm{~s}^{-1}$. Assuming continued expansion at this velocity, the shock will encounter the inner optical ring in the year $2006 \pm 3$. 
4. The eastern and western regions of the remnant align with the major axis of the optical ring, and are brighter than the northern and southern regions.

5. The eastern limb is brighter than the western limb, and is also increasing in brightness more rapidly.

6. Material on the eastern side of the remnant may have travelled further from the explosion than the rest of the remnant.

We propose the following basic theory. Points 1,2 and 3 above are consistent with the shock reaching a sudden increase in the density of the CSM. This is believed to be either the termination shock of the BSG wind, or the contact discontinuity to the RSG wind. The increased density and distance from the star of the latter region can perhaps better explain the observations.

Point 4 is good evidence that the shocked wind is axisymmetric in its shape and/or density distribution. That deceleration of a remnant seems coupled with distortion of its morphology (cf. SN 1993J) is also a good indication that the CSM is probably the cause for the observed structure. We expect that as the shock further expands into this axisymmetric CSM, the polar regions will elongate and eventually brighten. If observations show these regions remaining faint and becoming retarded in their expansion, an alternative explanation could be a toroidal distribution of ejecta.

Points 5 and 6 can be explained by the shock on the eastern side of the remnant reaching the density jump first. We propose that this may indicate a directional anisotropy in the SN explosion.

Monitoring and imaging SN 1987A with the ATCA will continue. In addition, the forthcoming upgrade of the ATCA to operation at frequencies up to $25 \mathrm{GHz}$ will allow diffraction-limited images of SN 1987A down to a resolution of $\sim 0^{\prime \prime} .4$. With such data, a better study of existing and new features will be possible. With resolved images at both 9 and $25 \mathrm{GHz}$, spectral index variations across the remnant will also be detectable, which may show differences in the energetics of hotspots and of the polar regions. For example, the brightest region of SN 1993J also appears to have the steepest spectrum (Marcaide et al. 1995b). 
It is not clear exactly what will happen to the radio emission when the shock reaches the optical ring - much will depend on the nature of the unshocked RSG wind and its density distribution, an issue on which current models are undecided. It is entirely possible that the appearance of the remnant once it has passed into this region will be quite different to what we see now. In any case, SN 1987A will likely become a spectacular object in all wavebands, and we will continue to observe it regularly in anticipation of this event.

We thank Lewis Ball, Dan Briggs, David Crawford, Bob Sault, Raylee Stathakis, Tony Turtle and Mark Walker for useful discussions and advice, and Chris Burrows and John Krist for their HST image of the optical nebula. We are also particularly grateful for the assistance, support and patience of the staff of the Paul Wild Observatory during the large number of observations for this project over the last seven years. BMG acknowledges the support of an Australian Postgraduate Award.

Requests for data should be directed via email to b.gaensler@physics.usyd.edu.au. 


\begin{tabular}{|c|c|c|c|c|c|c|}
\hline Epoch & $\begin{array}{l}\text { Date of } \\
\text { Observation }\end{array}$ & Day No. & $\begin{array}{c}\text { Integration } \\
\text { Time }\end{array}$ & $\begin{array}{c}\text { Diffraction } \\
\text { Limited Beam }\end{array}$ & $\begin{array}{l}\text { RMS Noise } \\
(\mu \mathrm{Jy} / \text { beam })\end{array}$ & $\begin{array}{c}\mathrm{S} \\
(\mathrm{mJy})\end{array}$ \\
\hline \multirow[t]{3}{*}{1992} & 21-Oct-1992 & 2068 & $17 \mathrm{~h}$ & $1^{\prime \prime} .11 \times 0 .^{\prime \prime} 85$ & 44 & $5.4 \pm 0.1$ \\
\hline & 04-Jan-1993 & 2142 & $15 \mathrm{~h}$ & $\mathrm{PA}=34^{\circ}$ & & \\
\hline & 05-Jan-1993 & 2143 & $7 \mathrm{~h}$ & & & \\
\hline \multirow[t]{3}{*}{1993} & 24-Jun-1993 & 2314 & $13 \mathrm{~h}$ & $0^{\prime \prime} .98 \times 0^{\prime \prime} .92$ & 34 & $6.8 \pm 0.1$ \\
\hline & 01-Jul-1993 & 2321 & $13 \mathrm{~h}$ & $\mathrm{PA}=12^{\circ}$ & & \\
\hline & 15-Oct-1993 & 2426 & $19 \mathrm{~h}$ & & & \\
\hline \multirow[t]{3}{*}{1994} & 16-Feb-1994 & 2550 & $13 \mathrm{~h}$ & $0^{\prime \prime} .99 \times 0^{\prime \prime} .94$ & 30 & $7.3 \pm 0.1$ \\
\hline & 27-Jun-1994 & 2683 & $26 h^{*}$ & $\mathrm{PA}=-78^{\circ}$ & & \\
\hline & 01-Jul-1994 & 2687 & $14 \mathrm{~h}$ & & & \\
\hline \multirow[t]{3}{*}{1995} & 24-Jul-1995 & 3074 & $11 \mathrm{~h}$ & $1^{\prime \prime} .04 \times 0^{\prime \prime} .89$ & 33 & $10.8 \pm 0.1$ \\
\hline & 29-Aug-1995 & 3111 & $11 \mathrm{~h}$ & $\mathrm{PA}=6^{\circ}$ & & \\
\hline & 06-Nov-1995 & 3178 & $12 \mathrm{~h}$ & & & \\
\hline
\end{tabular}

*spread over two days

Table 1: Imaging observations of SN 1987A with ATCA. Position angles are those of the major axis of the beam, and are measured north through east. 


\section{REFERENCES}

Ball, L., Campbell-Wilson, D., Crawford, D. F., \& Turtle, A. J. 1995, ApJ, 453, 864.

Ball, L., Campbell-Wilson, D., \& Staveley-Smith, L. 1995, MNRAS, 276, 944.

Ball, L. \& Kirk, J. G. 1992, ApJ, 396, L39.

Ball, L. \& Kirk, J. G. 1995, A\&A, 303, L57.

Bartel, N. et al. 1994, Nature, 368, 610.

Bartel, N., Rupen, M. P., Shapiro, I. I., Preston, R. A., \& Rius, A. 1991, Nature, 350, 212.

Beuermann, K., Brandt, S., \& Pietsch, W. 1994, A\&A, 281, L45.

Bisnovatyi-Kogan, G. S. 1971, Soviet Astron., 14, 652.

Blondin, J. M. 1994, in Circumstellar Media in the Late Stages of Stellar Evolution, ed. R. E. S. Clegg, W. P. S. Meikle, \& I. R. Stevens, Cambridge University Press, p. 139.

Blondin, J. M. \& Lundqvist, P. 1993, ApJ, 405, 337.

Blondin, J. M., Lundqvist, P., \& Chevalier, R. A. 1996, ApJ, . in press.

Bodenheimer, P. \& Woosley, S. E. 1983, ApJ, 269, 281.

Braun, R. \& Strom, R. G. 1986, A\&A, 164, 193.

Briggs, D. S. 1994, in The Restoration of HST Images and Spectra II, ed. R. J. Hanisch \& R. L. White, Space Telescope Science Institute, p. 250.

Briggs, D. S. 1995. PhD thesis, New Mexico Institute of Mining and Technology.

Burderi, L. \& King, A. R. 1995, MNRAS, 276, 1141.

Burrows, C. J. et al. 1995, ApJ, 452, 680. 
Chevalier, R. A. 1982, ApJ, 259, 302.

Chevalier, R. A. 1992a, Nature, 360, 628.

Chevalier, R. A. 1992b, Nature, 355, 617.

Chevalier, R. A. \& Dwarkadas, V. V. 1995, ApJ, 452, L45. (CD95).

Chevalier, R. A. \& Fransson, C. 1987, Nature, 328, 44.

Chevalier, R. A. \& Luo, D. 1994, ApJ, 421, 225.

Clark, B. G. 1980, A\&A, 89, 377.

Cropper, M., Bailey, J., McCowage, J., Cannon, R. D., Couch, W. J., Walsh, J. R., Strade, J. O., \& Freeman, F. 1988, MNRAS, 231, 695.

Crotts, A. P. S. \& Heathcote, S. R. 1991, Nature, 350, 683.

Crotts, A. P. S., Kunkel, W. E., \& Heathcote, S. R. 1995, ApJ, 438, 724.

Duffy, P., Ball, L., \& Kirk, J. G. 1995, ApJ, 447, 364. (DBK).

Frater, R. H., Brooks, J. W., \& Whiteoak, J. B. 1992, J. Electr. Electron. Eng. Aust., 12, 103.

Fulbright, M. S. \& Reynolds, S. P. 1990, ApJ, 357, 591.

Gorenstein, P., Hughes, J. P., \& Tucker, W. H. 1994, ApJ, 420, L25.

Gottesman, S. T., Broderick, J. J., Brown, R. L., Balick, B., \& Palmer, P. 1972, ApJ, 174, 383.

Gull, S. F. \& Daniell, G. J. 1978, Nature, 272, 686.

Hanuschik, R. W. \& Dachs, J. 1987, A\&A, 182, L29.

Hasinger, G., Aschenbach, B., \& Trümper, J. 1996, A\&A, 312, L9.

Jacobsen, P. et al. 1991, ApJ, 369, L63. 
Karovska, M., Koechlin, L., Nisenson, P., Papaliolios, C., \& Standley, C. 1989, in Highlights of Astronomy, Volume 8, ed. D. McNally, (Dordrecht: Kluwer), p. 193.

Kaspi, V. M., Bailes, M., Manchester, R. N., Stappers, B. W., \& Bell, J. F. 1996, Nature, 381, 584 .

Kesteven, M. J. \& Caswell, J. L. 1987, A\&A, 183, 118.

Landecker, T. L., Pineault, S., Routledge, D., \& Vaneldik, J. F. 1982, ApJ, 261, L41.

Lloyd, H. M., O’Brien, T. J., \& Kahn, F. D. 1995, MNRAS, 273, L19.

Luo, D. \& McCray, R. 1991, ApJ, 379, 659.

Luo, D., McCray, R., \& Slavin, J. 1994, ApJ, 430, 264.

Manchester, R. N. 1987, A\&A, 171, 205.

Manchester, R. N. 1988, Proc. Astron. Soc. Austral., 7, 548.

Manchester, R. N. \& Peterson, B. A. 1996, ApJ, 456, L107.

Marcaide, J. M. et al. 1995a, Nature, 373, 44.

Marcaide, J. M. et al. 1995b, Sci, 270, 1475.

Martin, C. L. \& Arnett, D. 1995, ApJ, 447, 378.

McCray, R. \& Lin, D. N. C. 1994, Nature, 369, 378.

Méndez, M., Clocchiatti, A., Benvenuto, O. G., Feinstein, C., \& Marraco, H. G. 1988, ApJ, 334, 295.

Narayan, R. \& Nityananda, R. 1986, ARA\&A, 24, 127.

Panagia, N., Scuderi, S., Gilmozzi, R., Challis, P. M., Garnavich, P. M., \& Kirshner, R. P. 1996, ApJ, 459, L17. 
Petre, R., Becker, C. M., \& Winkler, P. F. 1996, ApJ, 465, L43.

Plait, P. C., Lundqvist, P., Chevalier, R. A., \& Kirshner, R. P. 1995, ApJ, 439, 730.

Podsiadlowski, P., Fabian, A. C., \& Stevens, I. R. 1991, Nature, 354, 43.

Pun, C. S. J., Kirshner, R. P., Challis, P., \& Garnavich, P. 1995, BAAS, 27, 1308.

Reynolds, J. E. 1994, ATNF Technical Document Series, 39.3040.

Reynolds, J. E. et al. 1995, A\&A, 304, 116.

Roger, R. S., Milne, D. K., Kesteven, M. J., Wellington, K. J., \& Haynes, R. F. 1988, ApJ, 332, 940.

Sault, R. J., Teuben, P. J., \& Wright, M. C. H. 1995, in Astronomical Data Analysis Software and Systems IV, ed. R. Shaw, H.E. Payne, \& J.J.E. Hayes, ASP Conference Series, Volume 77, p. 433.

Sault, R. J. \& Wieringa, M. H. 1994, A\&AS, 108, 585.

Stathakis, R. A. 1996. PhD thesis, University of Sydney.

Staveley-Smith, L., Briggs, D. S., Rowe, A. C. H., Manchester, R. N., Reynolds, J. E., Tzioumis, A. K., \& Kesteven, M. J. 1993a, Nature, 366, 136. (Paper III).

Staveley-Smith, L. et al. 1992, Nature, 355, 147. (Paper I).

Staveley-Smith, L., Manchester, R. N., Kesteven, M. J., Tzioumis, A. K., \& Reynolds, J. E. 1993b, Proc. Astron. Soc. Austral., 10, 331. (Paper II).

Staveley-Smith, L., Manchester, R. N., Tzioumis, A. K., Reynolds, J. E., \& Briggs, D. S. 1996, in IAU Colloquium 145: Supernovae and supernova remnants, ed. R. McCray \& Z. Wang, Cambridge University Press, p. 309, (Paper IV).

Storey, M. C. \& Manchester, R. N. 1987, Nature, 329, 421. 
Storey, M. C., Staveley-Smith, L., Manchester, R. N., \& Kesteven, M. J. 1992, A\&A, 265, 752.

Suzuki, T., Shigeyama, T., \& Nomoto, K. 1993, A\&A, 274, 883.

Tuohy, I. R., Clark, D. H., \& Burton, W. B. 1982, ApJ, 260, L65.

Turtle, A. J. et al. 1987, Nature, 327, 38.

Utrobin, V. P., Chugai, N. N., \& Andronova, A. A. 1995, A\&A, 295, 129.

Walker, A. R. \& Suntzeff, N. B. 1990, PASP, 102, 131.

Wang, L. \& Mazzali, P. A. 1992, Nature, 355, 58.

Weiler, K. W., Sramek, R. A., Panagia, N., van der Hulst, J. M., \& Salvati, M. 1986, ApJ, 301, 790.

Weiler, K. W., Van Dyk, S. D., Sramek, R. A., \& Panagia, N. 1996, in IAU Colloquium 145: Supernovae and supernova remnants, ed. R. McCray \& Z. Wang, Cambridge University Press, p. 283.

Whiteoak, J. B. Z. \& Green, A. J. 1996, A\&AS, 118, 329.

Wilkinson, P. N. \& de Bruyn, A. G. 1990, MNRAS, 242, 529.

Willingale, R., West, R. G., Pye, J. P., \& Stewart, G. C. 1996, MNRAS, 278, 749. 


\section{FIGURE CAPTIONS}

Figure 1 : Light curves for the second radio turn-on of SN 1987A at four different frequencies. Where error bars are not shown, they are smaller than the plotted points.

Figure 2 : The radio spectrum of SN 1987A at five different epochs. The day number corresponds to the number of days since the explosion, and all observations were made within three days of the day number on each plot. Where available, $0.843 \mathrm{GHz}$ MOST data (Ball et al. 1995) have been included. The spectral index $\alpha\left(S \propto \nu^{\alpha}\right)$ has been calculated using a linear least-squares fit. Errors are no larger than the size of the plotted points.

Figure 3 : Diffraction-limited (top) and super-resolved images (bottom) of SN 1987A at $9 \mathrm{GHz}$, using data from epoch 1992 (see Table 1). An ellipse corresponding to the FWHM of the Gaussian restoring beam is shown at bottom right of each image. For the diffraction-limited image, the greyscale runs from -0.1 to $5.0 \mathrm{mJy}_{\mathrm{beam}}{ }^{-1}$, and the contours run from 0.5 to $5.0 \mathrm{mJy} \mathrm{beam}^{-1}$ at $0.5 \mathrm{mJy}$ beam $^{-1}$ intervals. For the super-resolved image, the greyscale is from -0.1 to $2.0 \mathrm{mJy}$ beam $^{-1}$, with contours from 0.2 to $2.0 \mathrm{mJy}^{\text {beam }}{ }^{-1}$ at $0.2 \mathrm{mJy}_{\text {beam }}{ }^{-1}$ intervals.

Figure 4: As in Figure 3, for 1993 data.

Figure 5: As in Figure [3, for 1994 data.

Figure 6: As in Figure 3, for 1995 data.

Figure 7 : A difference map for SN 1987A between 1993 and 1995. The greyscale corresponds to the super-resolved 1995 epoch data for SN 1987A (as in Figure 6), and ranges from -0.1 to $2.0 \mathrm{mJy}$ beam $^{-1}$. The contours correspond to a difference image between the 1995 and 1993 super-resolved data. Broken contour levels are -0.2 and -0.1 mJy beam, ${ }^{-1}$ while solid levels are 0.1 to $0.9 \mathrm{mJy}$ beam $^{-1}$ in increments of $0.1 \mathrm{mJy}_{\text {beam }}{ }^{-1}$.

Figure 8: Diffraction-limited and super-resolved images of 0536-6919 at $9 \mathrm{GHz}$, using data from epoch 1995. For the diffraction-limited image, the greyscale runs from -0.1 to $6.0 \mathrm{mJy}$ beam $^{-1}$, and the contours run from 0.5 to $6.0 \mathrm{mJy}^{\text {beam }^{-1}}$ at $0.5 \mathrm{mJy}^{\mathrm{beam}}{ }^{-1}$ intervals. For the 
super-resolved image, the greyscale is from -0.1 to $5.0 \mathrm{mJy}^{\text {beam }}{ }^{-1}$, with contours from 0.5 to 5.0 mJy beam ${ }^{-1}$ at $0.5 \mathrm{mJy}_{\text {beam }}^{-1}$ intervals.

Figure 9 : A comparison of HST and ATCA images of SN 1987A. Contours show the 1995 epoch of the super-resolved ATCA $9 \mathrm{GHz}$ data. Contours run from 0.2 to $2.0 \mathrm{mJy}^{\mathrm{beam}}{ }^{-1}$ at $0.2 \mathrm{mJy}$ beam ${ }^{-1}$ increments. At bottom right is shown the $0^{\prime \prime} .4 \mathrm{FWHM}$ restoring beam. The greyscale is a WFPC2 HST image of SN 1987A in $\mathrm{H} \alpha+$ [NiI] (Burrows et al. 1995), to which the reference frame of Reynolds et al. (1995) has been applied. The point source at the lower left of the HST image is an unrelated field star (star 3 of Walker \& Suntzeff 1990).

Figure 10 : The radius of the radio remnant as a function of time, as determined by fitting a thin spherical shell of arbitrary position, flux density and radius to the $u-v$ data for $9 \mathrm{GHz}$ observations in Paper III and in Table 1. The broken line is a power-law fit to the data of the form $r \propto t^{m}$, with $m=0.12$. The solid line is a linear fit to the data, with slope $32 \mu$ as day ${ }^{-1}$ and with $r=0^{\prime \prime} .642$ at $t=1800$ days. 


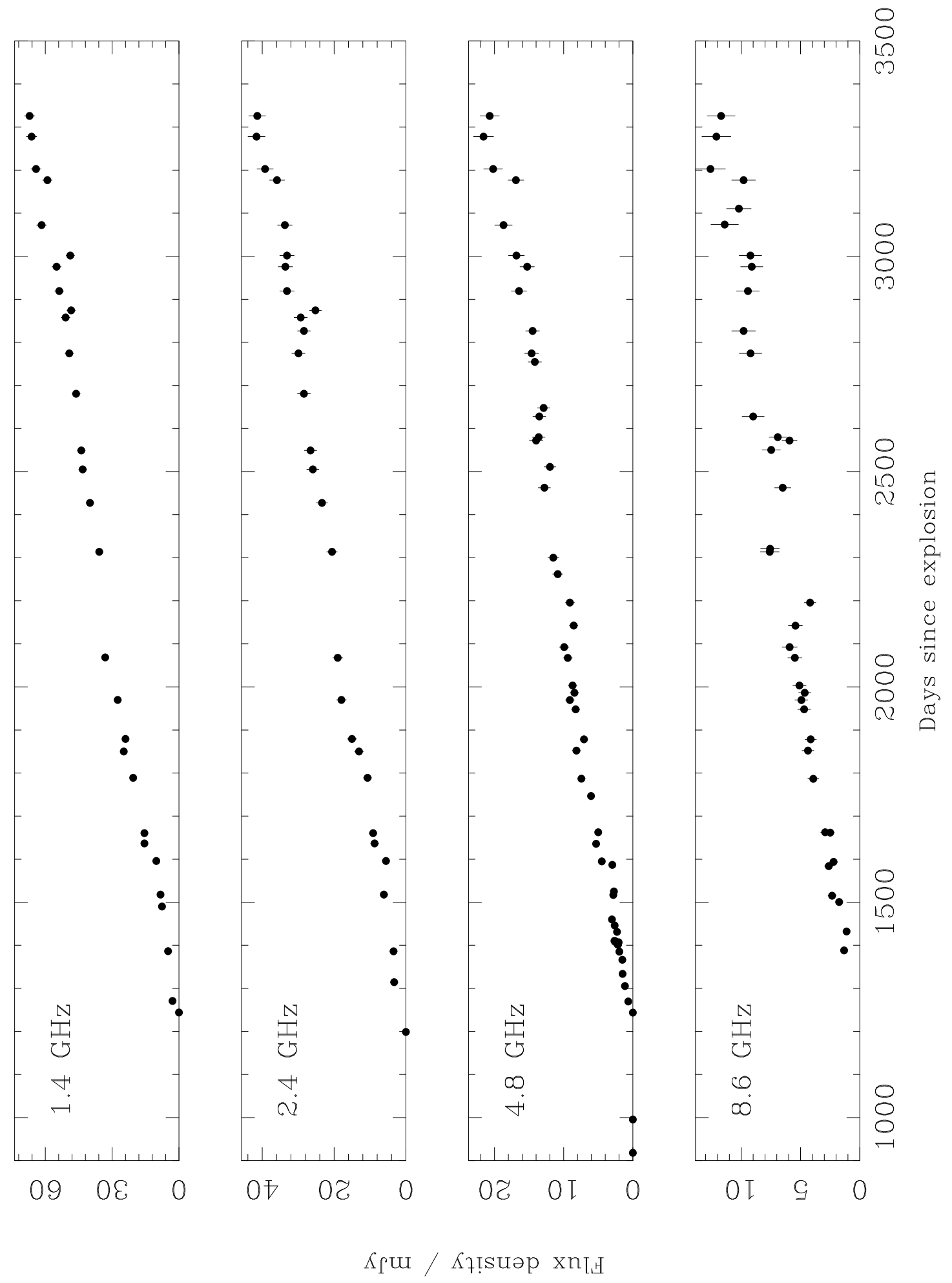

Fig. 1.- 


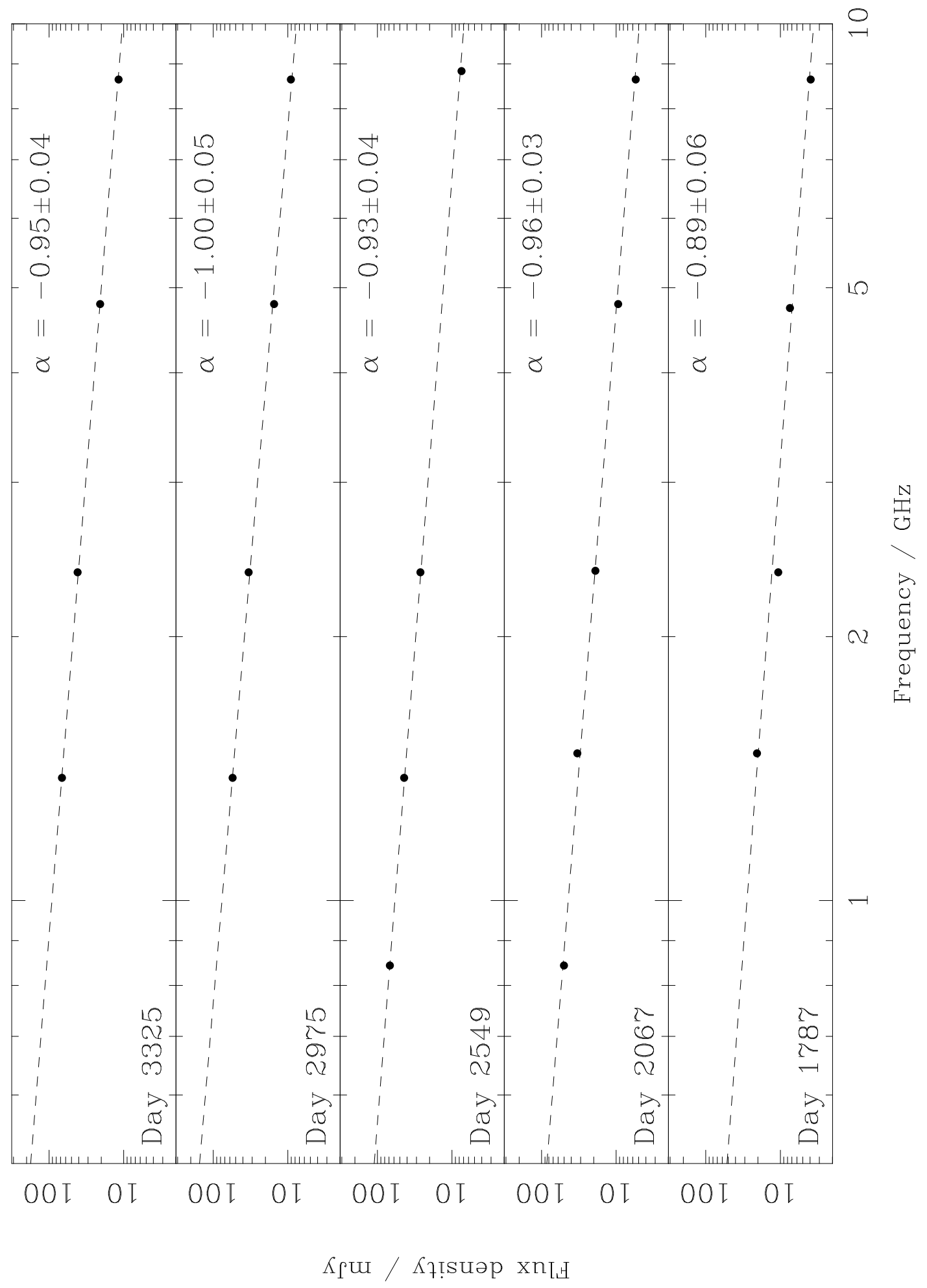

Fig. 2.- 

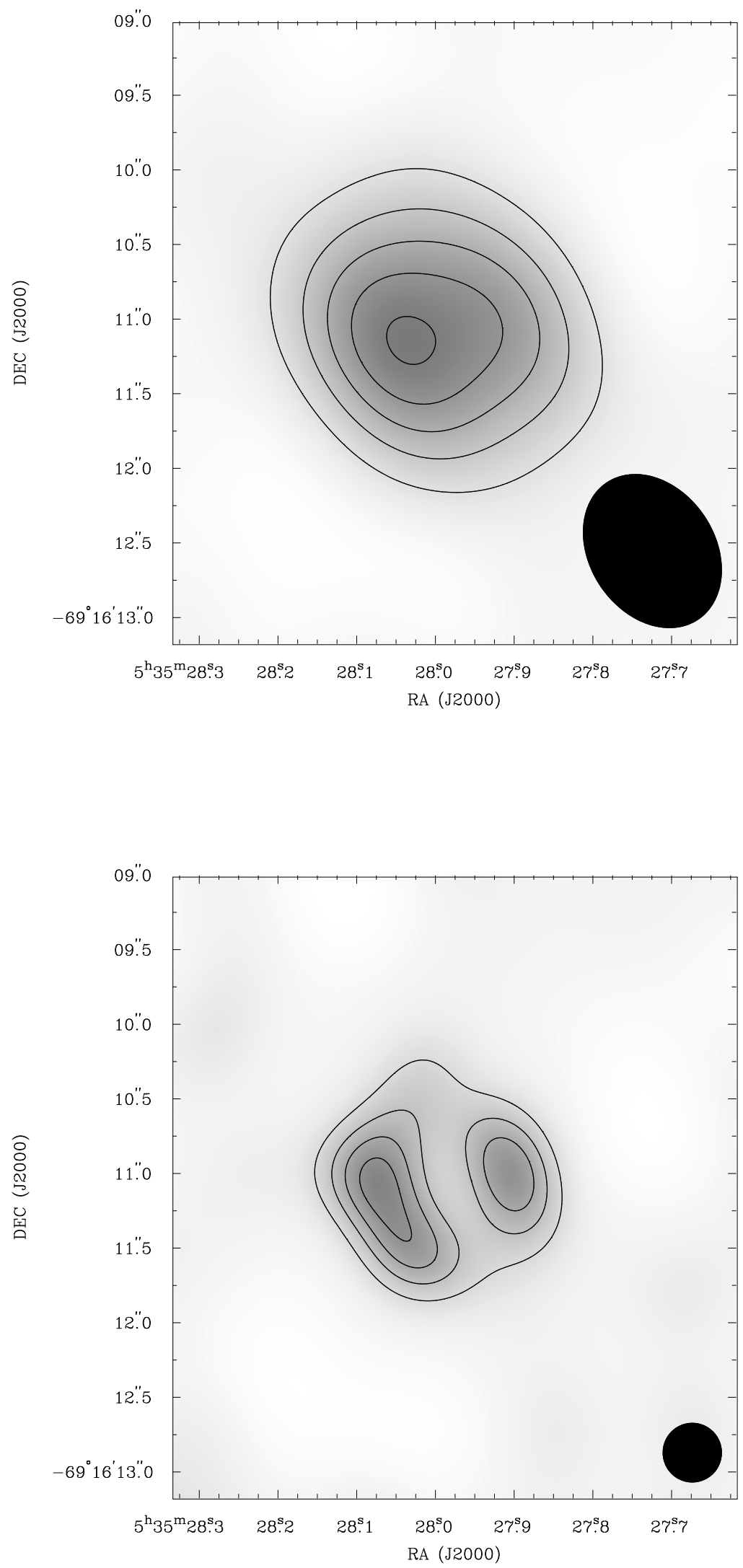

Fig. 3.- 

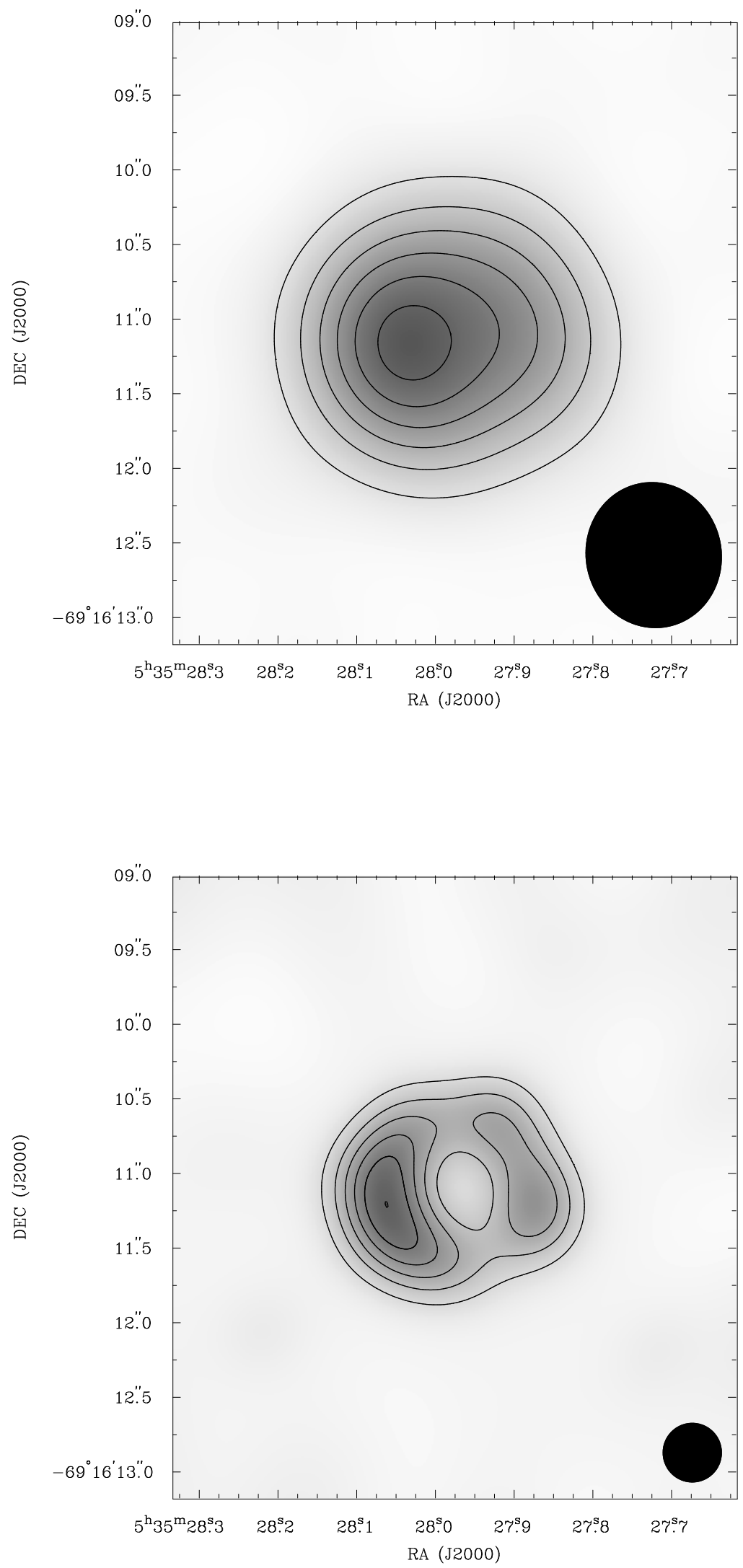

Fig. 4.- 

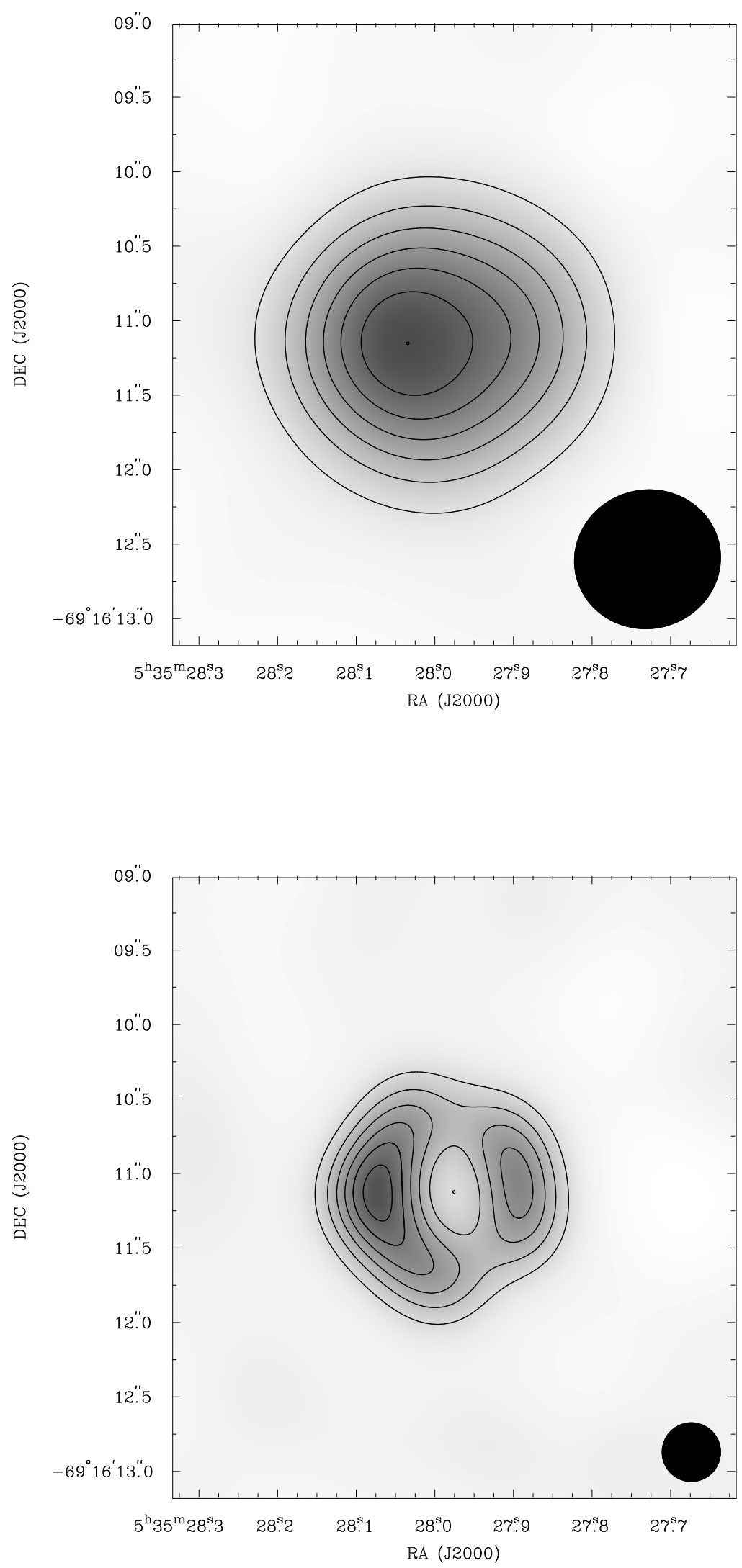

Fig. 5.- 

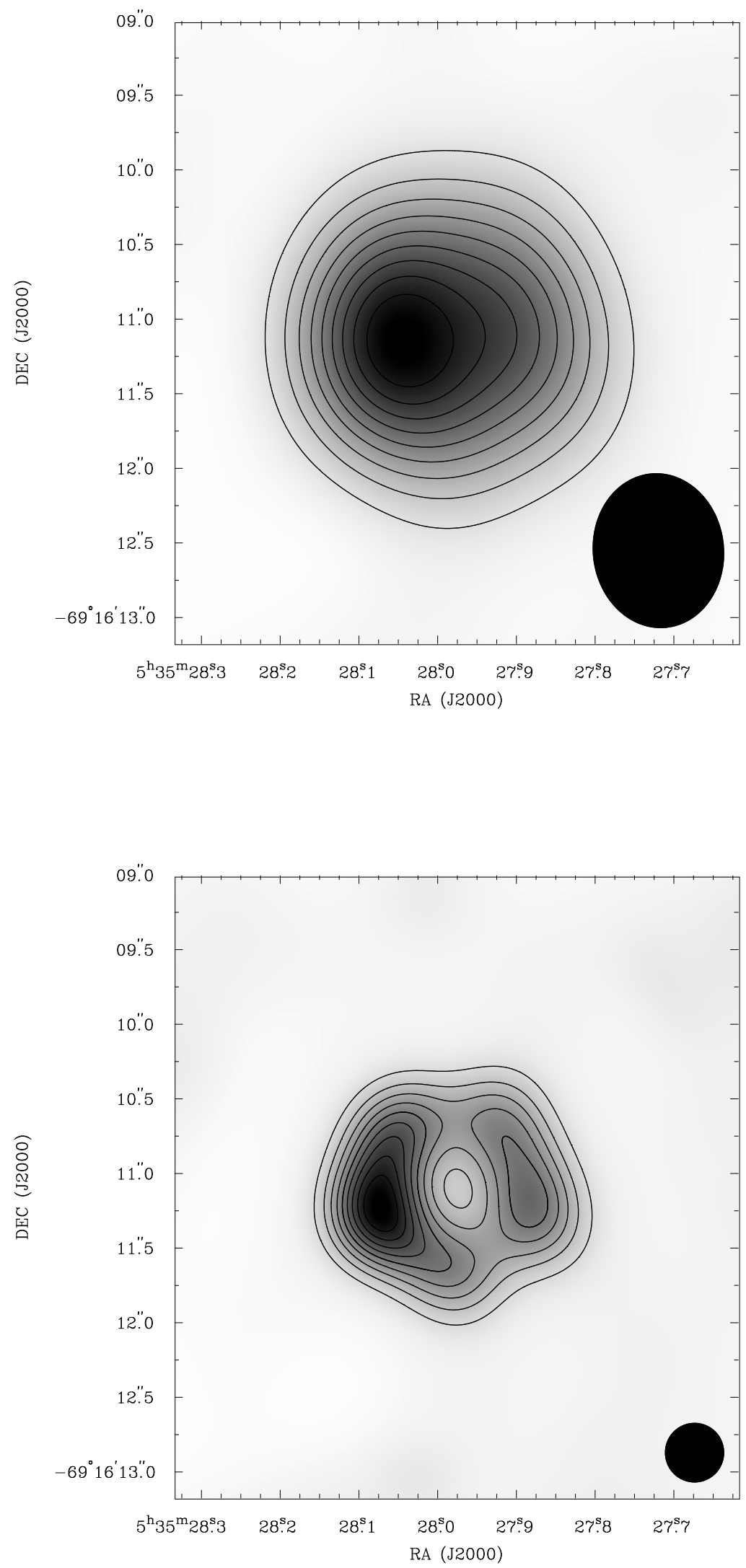

Fig. 6.- 


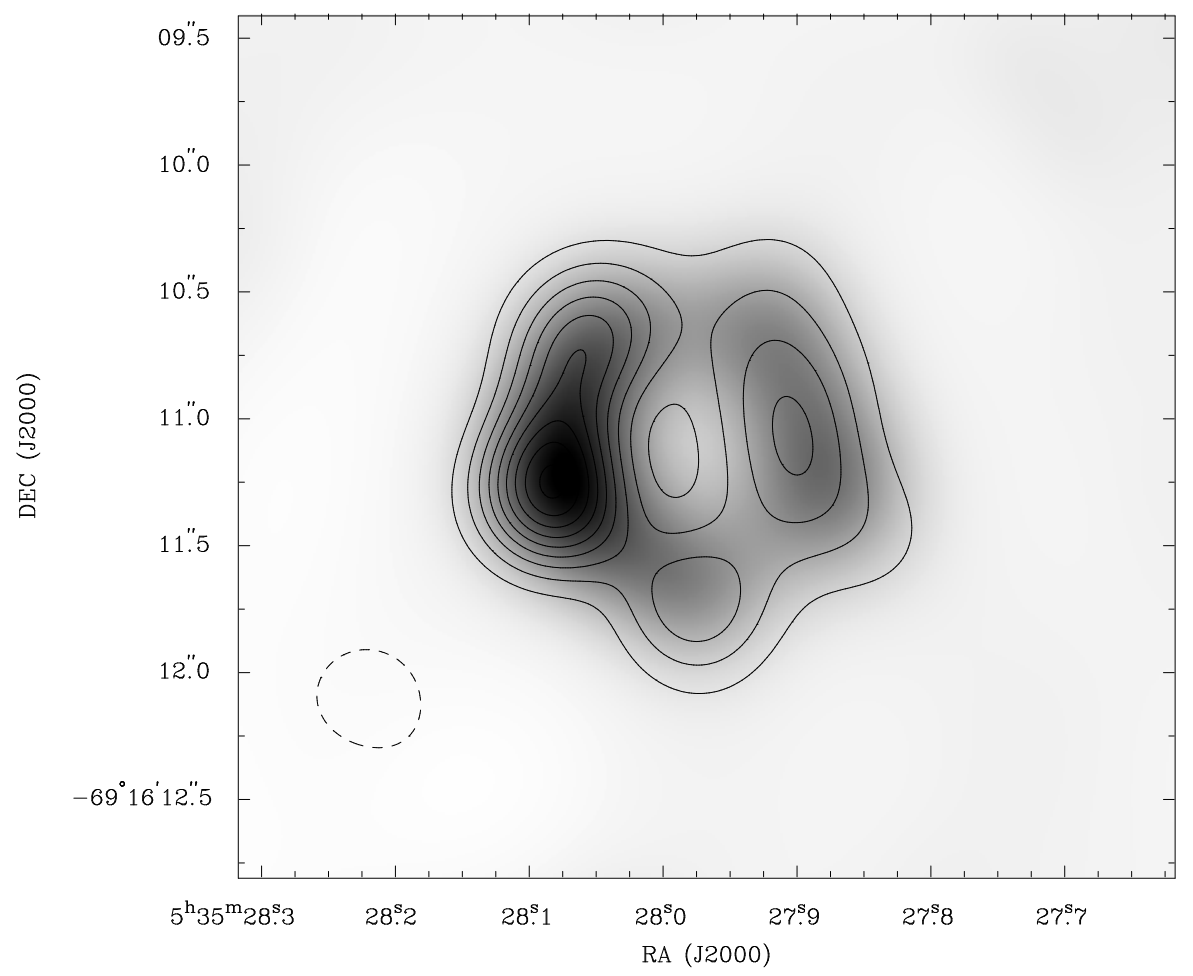

Fig. 7.- 

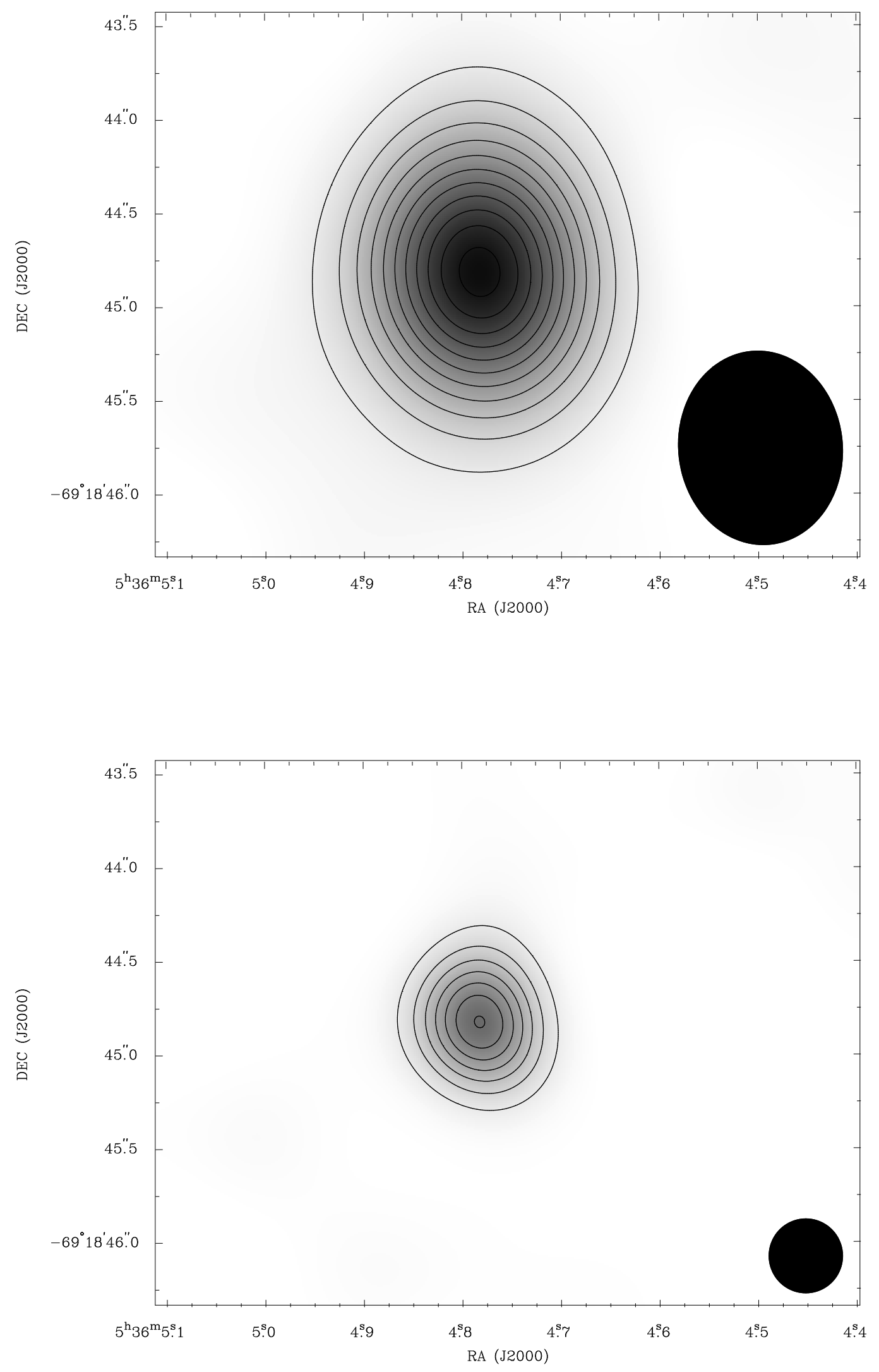

Fig. 8.- 


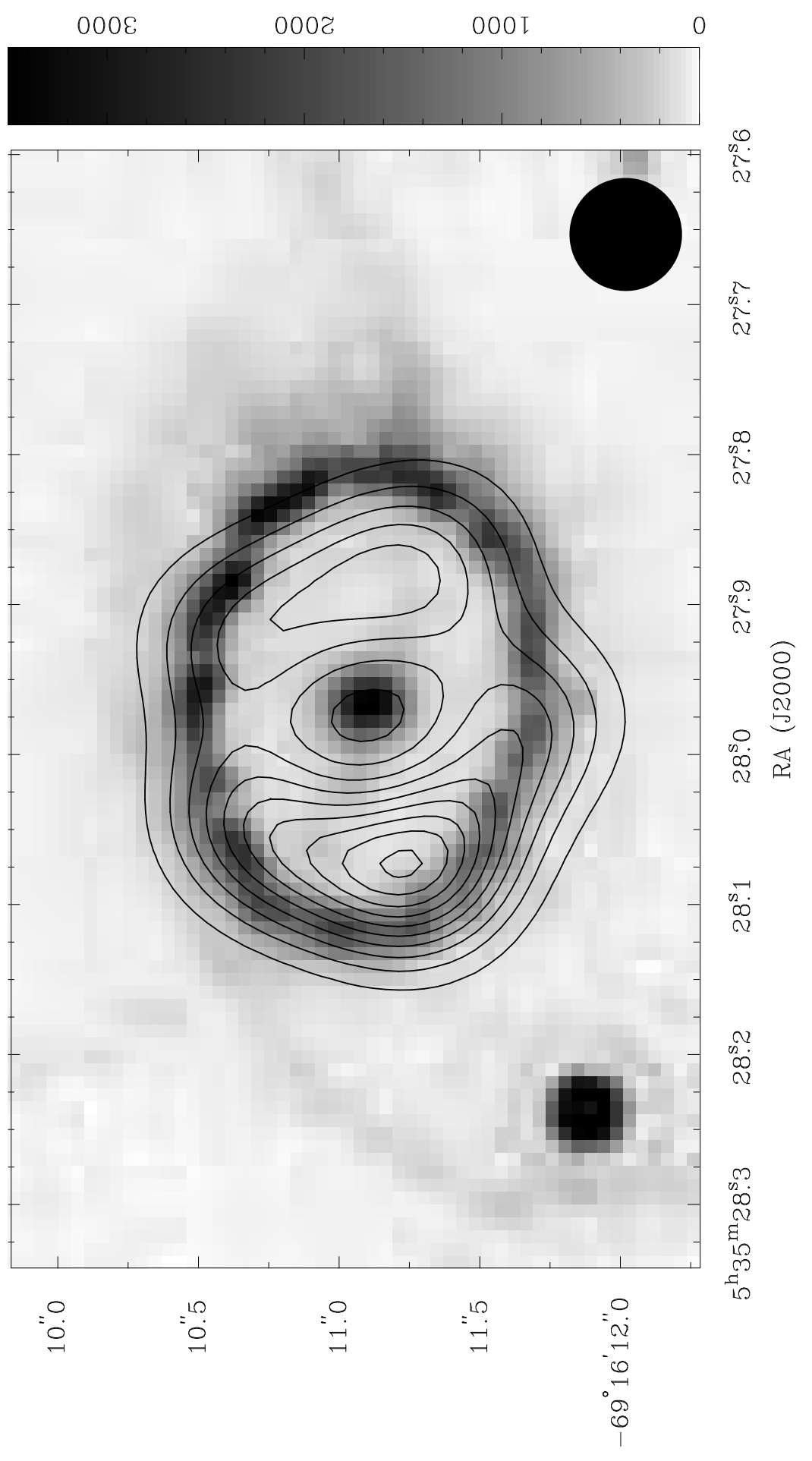

(000z) ) DHC

Fig. 9.- 


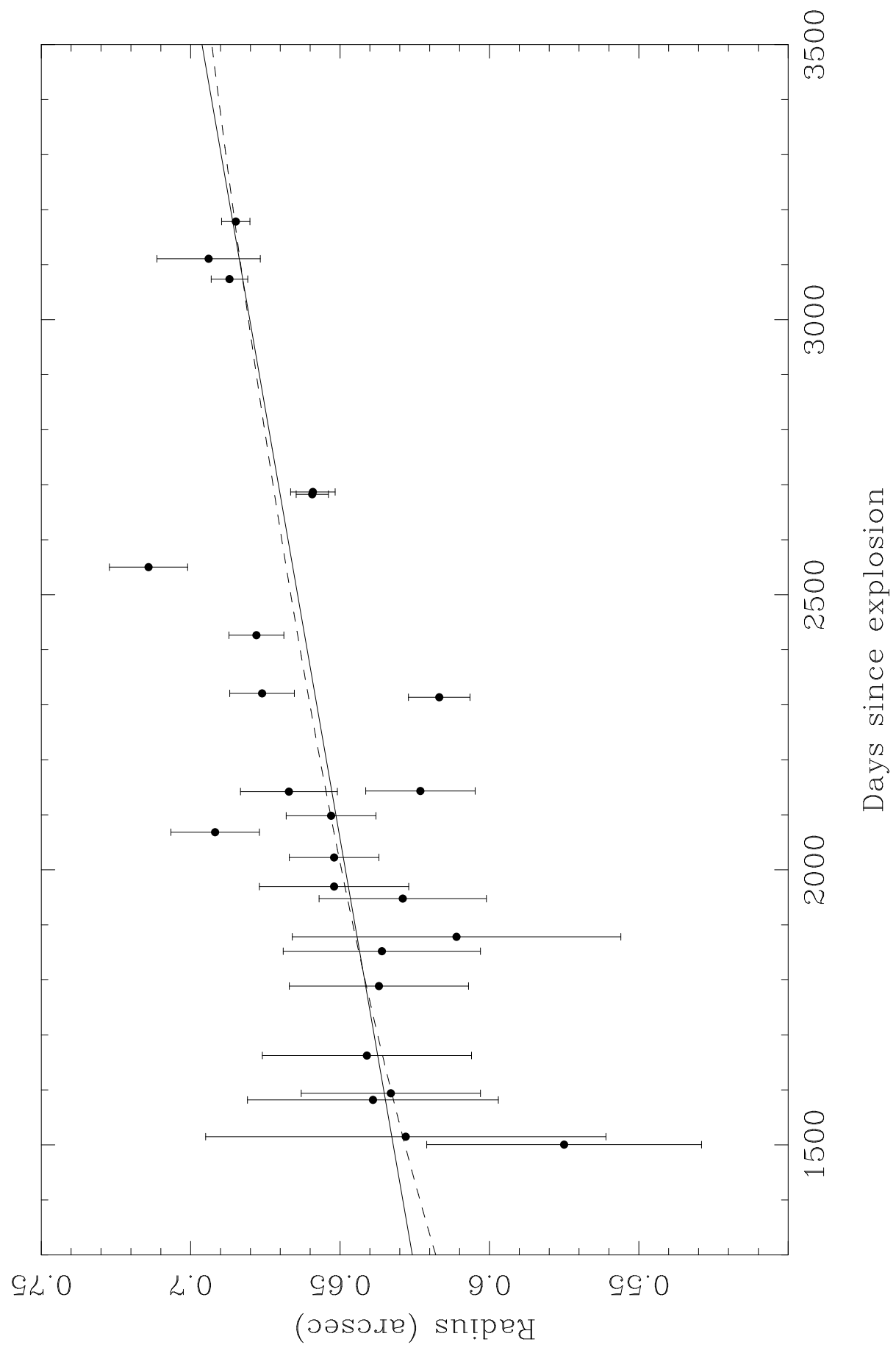

Fig. 10.- 